\title{
Combining the mammalian target of rapamycin inhibitor, rapamycin, with resveratrol has a synergistic effect in multiple myeloma
}

\author{
HONG-GUANG JIN ${ }^{1}$, GUO-ZHEN WU ${ }^{2}$, GUO-HUA WU ${ }^{1}$ and YONG-GE BAO ${ }^{1}$ \\ ${ }^{1}$ Department of Orthopedics, Affiliated Hospital of Inner Mongolia University for The Nationalities, \\ Tongliao, Inner Mongolia 028007; ${ }^{2}$ Department of Cardiology, Tongliao Traditional Chinese Medicine Hospital, \\ Tongliao, Inner Mongolia 028000, P.R. China
}

Received November 7, 2016; Accepted January 24, 2018

DOI: $10.3892 / \mathrm{ol} .2018 .8178$

\begin{abstract}
Rapamycin is known to inhibit the mammalian target of rapamycin complex (mTORC)1 signaling pathway, but it is unable to effectively inhibit mTORC2, resulting in activation of protein kinase $B$ in multiple myeloma (MM) cell lines. Additionally, certain studies have suggested that resveratrol has an effect on human MM cells, and that rapamycin in combination with resveratrol may be useful in cancer therapy. The present study aimed to investigate the combined treatment effect of resveratrol and rapamycin on the MM MM1.S cell line. The results demonstrated that combined treatment with rapamycin and resveratrol effectively inhibited cell viability in the MM1.S cell line through inhibition of the mTORC1 and mTORC2 signaling pathways, compared with resveratrol or rapamycin monotherapy. In addition, cyclin D1 levels were decreased and the activation of caspase- 3 and poly (ADP-ribose) polymerase was increased. These results suggested that downregulation of the mTOR signaling cascades is likely to be a crucial mediator in the impairment of viability and the induction of apoptosis resulting from combined therapy with resveratrol and rapamycin in MM1.S cells.
\end{abstract}

\section{Introduction}

Multiple myeloma (MM) is characterized by the accumulation of malignant plasma cells within the bone marrow (BM) and is the second most common type of hematological cancer, accounting for $10-15 \%$ of all types of hematological cancer. At present, chemotherapy is one of the primary treatments for MM, including newly marketed proteasome inhibitors and

Correspondence to: Professor Yong-Ge Bao, Department of Orthopedics, Affiliated Hospital of Inner Mongolia University for The Nationalities, 1742 Holin He Street, Tongliao, Inner Mongolia 028007, P.R. China

E-mail: baoyongge001@163.com

Key words: rapamycin, resveratrol, multiple myeloma, MM1.S cells immunomodulators. However, MM remains incurable in the majority of patients, with a median survival time of 3-5 years with conventional treatments. All current drugs exhibit limited efficacy and/or severe toxicity or adverse effects (1-4). Therefore, novel agents and combinations are required to further improve the survival of patients with MM.

Previous studies have indicated that the phosphoinositide 3-kinase (PI3K)/protein kinase B (Akt) signaling pathway and mammalian target of rapamycin (mTOR) are aberrantly activated in myeloma cells $(5,6)$. mTOR, a serine (Ser)/threonine (Thr) protein kinase expressed in the PI3K pathway, is not only a possible valuable target for the treatment of MM (7), but also has a vital function in regulating critical cellular processes, including transcription, ribosomal biogenesis, protein synthesis and cytoskeletal organization. In particular, mTOR coordinates cell viability in response to inputs of growth factors, energy stress and nutrient status, in addition to regulating cell cycle progression and survival (8-10). In mammalian cells, mTOR, via its two distinct multiprotein complexes, mTORC1 and mTORC2, has an important function in the regulation of different biological functions. $\mathrm{mTORC} 1$ is sensitive to rapamycin and phosphorylates its downstream effectors, including p70 S6 kinase, which in turn phosphorylates ribosomal S6 protein, thereby promoting protein biosynthesis. Activated mTORC1 also phosphorylates eukaryotic translation initiation factor 4E-binding protein 1 and promotes the formation of the protein synthesis initiation complex (11). mTORC2, however, regulates the growth, proliferation and angiogenesis of cancer cells, as a result of its contribution to the phosphorylation of Akt at the $\mathrm{Ser}^{473}$ residue, which is crucial for the maximal activation of Akt (12). In addition, mTORC1 is able to negatively influence Akt activity by inhibiting mTORC2 (13-15). Furthermore, the mTORC has a well-known function in MM cell proliferation, tumor development and chemoresistance through the diverse actions of mTORC1 and mTORC 2 and, as such, a disturbance of the delicate balance of this pathway may lead to MM cell apoptosis, tumor regression and an extended survival time for patients with MM (16). Therefore, inhibitors of the mTOR pathway may be promising future treatment options for MM.

The majority of studies regarding the mTOR pathway in cancer treatment primarily focus on rapamycin and its 
derivatives, which are known to selectively inhibit mTORC1 and to upregulate the Akt and extracellular signal-regulated kinase (ERK)/mitogen-activated protein kinase (MAPK) pathways via a PI3K-dependent feedback loop, leading to enhanced cell survival and resistance to chemotherapy $(17,18)$. mTORC2 was previously believed to phosphorylate Akt at $\mathrm{Ser}^{473}$ and to be the mediator of rapamycin-associated drug resistance. In contrast, in a previous study, mTORC 2 inhibition by rapamycin did not prevent increased phosphorylation of Akt or drug resistance (19). Due to the fact that mTORC2 was considered to phosphorylate Akt at $\mathrm{Ser}^{473}$, it was initially understood to be the mediator of rapamycin-associated drug resistance. Based upon existing data, it appears that the efficacy of monotherapy with rapamycin or its derivatives in the treatment of MM is highly limited (7). This may be due to the fact that rapamycin and its derivatives do not completely block mTORC1 and are unable to effectively inhibit mTORC2 to trigger feedback activation of Akt signaling in order to attenuate antitumor activity $(20,21)$. In recent years, preclinical data have demonstrated that mTOR inhibitors, including rapamycin, temsirolimus and everolimus, may be potential target therapies for the treatment of MM, particularly if combined with other drugs $(22,23)$. Therefore, it is reasonable to hypothesize that combined therapy with rapamycin and novel naturally-occurring compounds that suppress the rapamycin-induced Akt/ERK activation may improve the therapeutic efficacy of rapamycin against a broad range of human tumor cells.

Integrative medicine presents the theory of utilizing traditional Western medical principles synergistically with other evidence-based biomedical concepts. Accordingly, resveratrol (3,4',5-trihydroxy-trans-stilbene; Fig. 1A), a polyphenolic compound that was first isolated in 1940 as an ingredient of the roots of white hellebore (Veratrum grandiflorum O.Loes), was selected for use in the present study. Since its isolation, resveratrol has been identified in extracts from $>70$ other plant species $(24,25)$, and demonstrates antitumor effects both in vivo and in vitro through regulation of cell division, growth, angiogenesis and metastasis (26). Additionally, resveratrol has been reported to inhibit the proliferation and induce the apoptosis of MM cells, as well as overcoming the chemoresistance of these cells $(27,28)$. In human ovarian cancer cells, resveratrol induces phosphatase and tensin homolog, in addition to reducing the levels of phosphorylated-Akt (p-Akt) and mTOR $(29,30)$. Furthermore, certain studies have suggested that resveratrol may be useful in cancer therapy when used in combination with rapamycin in the treatment of breast cancer and chronic myeloid leukemia, primarily due to its ability to suppress the PI3K/Akt/mTOR signaling pathway $(31,32)$. However, to the best of our knowledge, whether or not MM may be treated by combined therapy with resveratrol and rapamycin has not previously been reported.

The aim of the present study was to investigate whether combining resveratrol with rapamycin has potential antitumor effects in a human MM cell line and to determine whether modulation of the PI3K/Akt/mTOR signaling pathway by resveratrol is crucial for its anticancer effects in a human MM cell line.

\section{Materials and methods}

MM cell lines and cell culture. Dexamethasone-sensitive MM1.S and doxorubicin-resistant RPMI-8226/DOX40 cell lines were obtained from the American Type Culture Collection (ATCC; Manassas, VA, USA). The two MM cell lines were cultured in RPMI-1640 medium (Sigma-Aldrich; Merck KGaA, Darmstadt, Germany), containing 10\% fetal bovine serum (FBS; Sigma-Aldrich; Merck KGaA), 2 mM L-glutamine (Gibco; Thermo Fisher Scientific, Inc., Waltham, MA, USA), $100 \mathrm{U} / \mathrm{ml}$ penicillin and $100 \mu \mathrm{g} / \mathrm{ml}$ streptomycin (both Gibco; Thermo Fisher Scientific, Inc.) at $37^{\circ} \mathrm{C}$ with $5 \% \mathrm{CO}_{2}$ in a humid incubator.

Reagents and antibodies. Resveratrol (Fig. 1A), dimethyl sulfoxide (DMSO), MTT and rapamycin were purchased from Sigma-Aldrich; Merck KGaA. Annexin V-fluorescein isothiocyanate and propidium iodide were purchased from BD Biosciences (San Jose, CA, USA). All primary antibodies were purchased from Cell Signaling Technology, Inc. (Danvers, MA, USA). The secondary horseradish peroxidase-conjugated mouse anti-rabbit IgG polyclonal antibodies for western blot analysis were provided by Beijing Zhongshan Golden Bridge Biotechnology Co., Ltd. (Beijing, China).

Cell viability assay. All MM cells were cultured for $24 \mathrm{~h}$ at $37^{\circ} \mathrm{C}$ in RPMI-1640 medium (Sigma-Aldrich; Merck KGaA) alone or with varying concentrations of rapamycin $(0,5,10,20$, 50 and $100 \mathrm{nM})$, resveratrol $(0,10,20,50,100$ and $200 \mu \mathrm{M})$ or a combination of the two drugs (concentrations of resveratrol and rapamycin were $60 \mu \mathrm{M}$ and $20 \mathrm{nM}$, respectively). In all the experiments, control wells were included with DMSO at the highest concentration tested with resveratrol or rapamycin. Cells $\left(1 \times 10^{4}\right)$ from 24 -h cultures were analyzed using an MTT assay. The medium was completely removed and $200 \mu \mathrm{l}$ DMSO was added to dissolve the MTT formazan crystals. Absorbance readings at a wavelength of $570 \mathrm{~nm}$ (OD570) were taken on a microplate reader (MQX 200; BioTek Instruments, Inc., Winooski, VT, USA). At least three independent experiments were performed.

Western blot analysis. For the analysis of mTORC1, mTORC2, caspase-3, poly ADP ribose polymerase (PARP), cyclin D1 and retinoblastoma protein $(\mathrm{Rb})$ expression, whole cells were lysed in ice-cold lysis buffer [50 mM Tris-HCl ( $\mathrm{pH} \mathrm{7.5),}$ $150 \mu \mathrm{M} \mathrm{NaCl}, 20 \mu \mathrm{M} \mathrm{NaF}, 20 \mu \mathrm{M}$ b-glycerophosphate, $1 \%$ Nonidet P-40, $1 \%$ Sodium Lauryl Sulfate, $10 \mathrm{mg} / \mathrm{ml}$ phenylmethanesulfonyl fluoride, $5 \mathrm{mM}$ EDTA, $10 \mathrm{mg} / \mathrm{ml}$ aprotinin and $10 \mathrm{mg} / \mathrm{ml}$ leupeptin]. Equal quantities of protein from total cell extracts were mixed with a sample buffer containing $100 \mathrm{mM} \beta$-mercaptoethanol and were heated for $10 \mathrm{~min}$ at $97^{\circ} \mathrm{C}$. Content of the total protein was detected by BCA protein assay kit (Tiangen Biotech Co., Ltd., Beijing, China). Protein $(20 \mu \mathrm{g})$ samples were separated by $10 \%$ SDS-PAGE and were transferred onto Immobilon polyvinylidene difluoride membranes (EMD Millipore, Billerica, MA, USA). Polyvinylidene difluoride membranes were incubated in 5\% non-fat dry milk at room temperature for $2 \mathrm{~h}$. Rabbit anti-mTORC1 (cat. no. 2587; 1:1,000), mouse anti-mTORC2 (cat. no. 13017; 1:1,000), rabbit anti-caspase-3 (cat. no. 9664; 1:1,000) and rabbit anti-PARP (cat. no. 9532; 1:1,000) antibodies (Cell Signaling Technology, Inc.) were added and incubated overnight at $4^{\circ} \mathrm{C}$. Peroxidase-conjugated Affinipure goat anti-rabbit IgG (cat. no. ZB-2301, dilution 1:5,000) and 
A<smiles>Oc1ccc(/C=C/c2cc(O)cc(O)c2)cc1</smiles>

Resveratrol

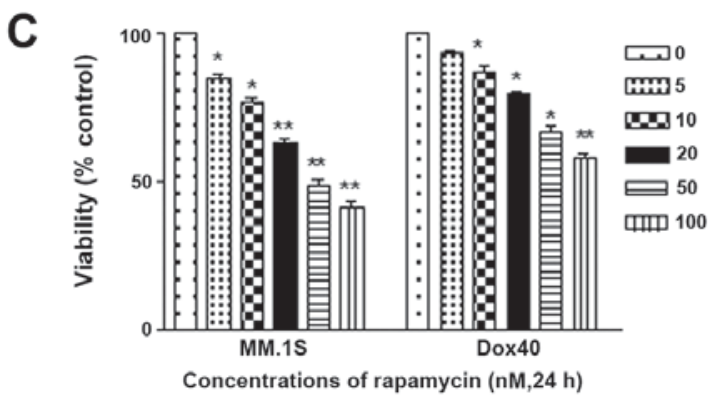

B

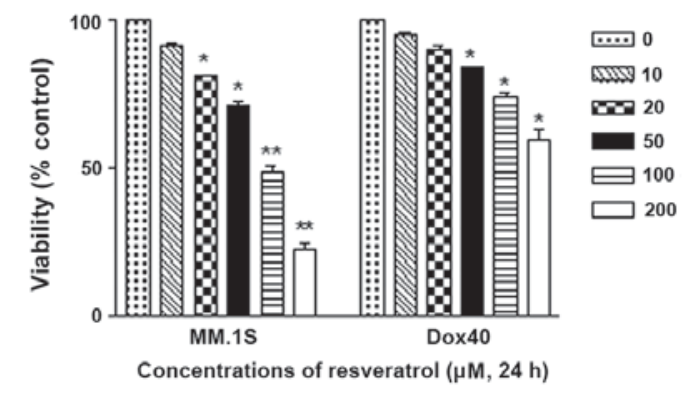

D

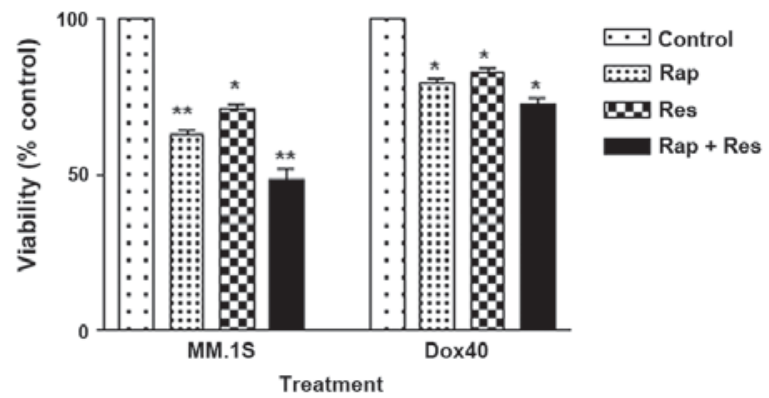

Figure 1. Resveratrol structure and resveratrol, rapamycin and combination treatment suppresses cell viability of MM cells. (A) Molecular structure of resveratrol. (B) Inhibitory effect of resveratrol on the viability of human MM cells. (C) Inhibitory effect of rapamycin on the viability of human MM cells. (D) Effect of resveratrol, rapamycin and their combination on MM cell viability. Cells were treated with dimethyl sulfoxide as a vehicle control or with resveratrol $(60 \mu \mathrm{M})$, rapamycin $(20 \mathrm{nM})$ or their combination [resveratrol $(60 \mu \mathrm{M})+$ rapamycin $(20 \mathrm{nM})$ ] for 24 h and cell viability was determined using an MTT assay. ${ }^{*} \mathrm{P}<0.05,{ }^{* *} \mathrm{P}<0.01$ vs. vehicle control. MM, multiple myeloma; Res, resveratrol; Rap, rapamycin.

rabbit anti-mouse IgG (cat. no. ZDR-5109, dilution 1:5,000) (Beijing Zhongshan Golden Bridge Biotechnology Co., Ltd.) were then added separately and incubated for $2 \mathrm{~h}$ at room temperature. Following washing, membranes were incubated with horseradish peroxidase-conjugated secondary antibodies at room temperature for $1 \mathrm{~h}$. Blots were then developed using an enhanced chemiluminescence kit (EMD Millipore). Densitometric analysis of protein bands was performed using Image J v.1.45s software (National Institutes of Health, Bethesda, MD, USA). All western blot analysis was performed $\geq 3$ times to ensure reproducibility.

Statistical analysis. Data are expressed as the mean \pm standard deviation. SPSS version 17.0 software (SPSS, Inc., Chicago, IL, USA) was used for statistical analysis. Comparisons among multiple groups were determined using one-way analysis of variance, followed by Fisher's least significant difference test (for equal variances assumed) or Dunnett's T3 test (for equal variances not assumed) to compare individual group differences. $\mathrm{P}<0.05$ was considered to indicate a statistically significant difference.

\section{Results}

Resveratrol, rapamycin and combined treatment suppress the viability of MM cells. In the present study, an MTT assay was used to determine the effect on cell viability of resveratrol and rapamycin. The MM MM1.S and RPMI-8226/DOX40 cell lines were cultured in complete medium in the absence (control) or presence of various concentrations of resveratrol and rapamycin for indicated time periods. Untreated cells were considered as the baseline (100\%) for analysis. The viability-impairing activity of resveratrol, rapamycin and the combination of the two is presented in Fig. 1B-D. Cells were initially treated with varying concentrations of resveratrol $(0,10,20,50,100$ and $200 \mu \mathrm{M})$ and rapamycin $(0,5,10,20,50$ and $100 \mathrm{nM})$ for $24 \mathrm{~h}$. Resveratrol and rapamycin significantly impaired the viability of the MM1.S cells in a dose-dependent manner, which was more significant than the effect on RPMI-8226/DOX40 cells. For the combined treatment, $60 \mu \mathrm{M}$ resveratrol was used with $20 \mathrm{nM}$ rapamycin. In the MM.1S cells, the reduction in the rate of cell viability proliferation was significantly increased in the combination treatment group compared with the control group $(\mathrm{P}<0.01)$, and in RPMI-8226/DOX40 cells there was a significant reduction in the rate of cell viability proliferation in the combination treatment group compared with the blank group $(\mathrm{P}<0.05)$. Therefore, the MM.1S cells were more sensitive to combination therapy compared with the RPMI-8226/DOX40 cells. Compared with resveratrol or rapamycin monotherapy, the combination of the two drugs was much more effective in impairing the viability of the MM1.S cells, an effect that was more notable than that on the RPMI-8226/DOX40 cells. These results indicated that combined drug therapy may improve the treatment of MM, as single-agent therapy is becoming less effective as a result of the multi-drug resistance exhibited by cancer cells.

mTOR_Akt signaling in MM cells. The present study initially examined whether or not autophosphorylation of mTOR at $\mathrm{Ser}^{2481}$ may be used as readout for mTORC2/Akt signaling in MM cells. Therefore, the baseline phosphorylation of mTOR on $\operatorname{Ser}^{2481}$ in MM cell lines was evaluated. Normally, mTORC1 primarily contains mTOR phosphorylated at 


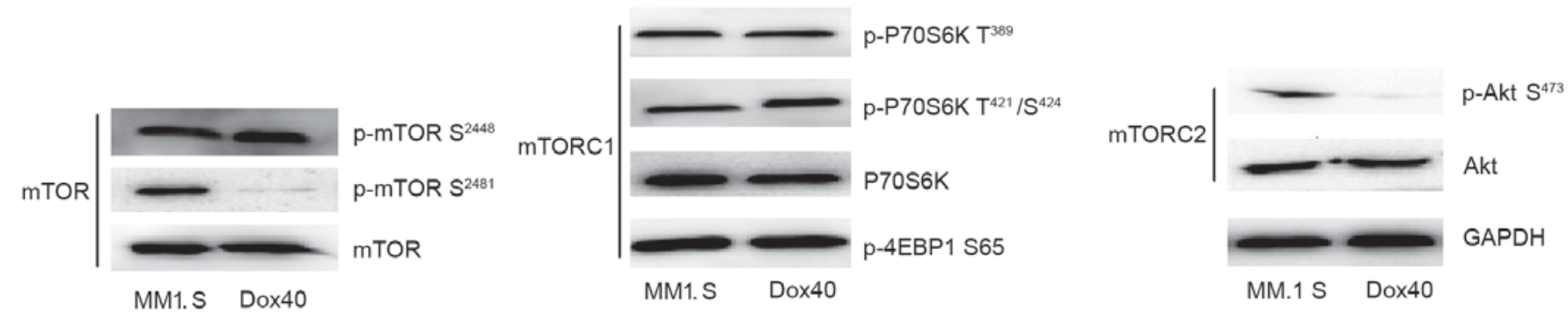

Figure 2. mTOR_Akt signaling in MM cells: Baseline mTOR profile in multiple myeloma cell lines. MM cells cultured in culture media alone were lysed and $\mathrm{mTOR} / \mathrm{mTORC} 1 / \mathrm{mTORC} 2$-associated protein expression was determined by western blot analysis. mTOR, mammalian target of rapamycin; mTORC, mammalian transcriptional coactivator for CREB; Dox40, RPMI-8226/Dox40; MM, multiple myeloma.
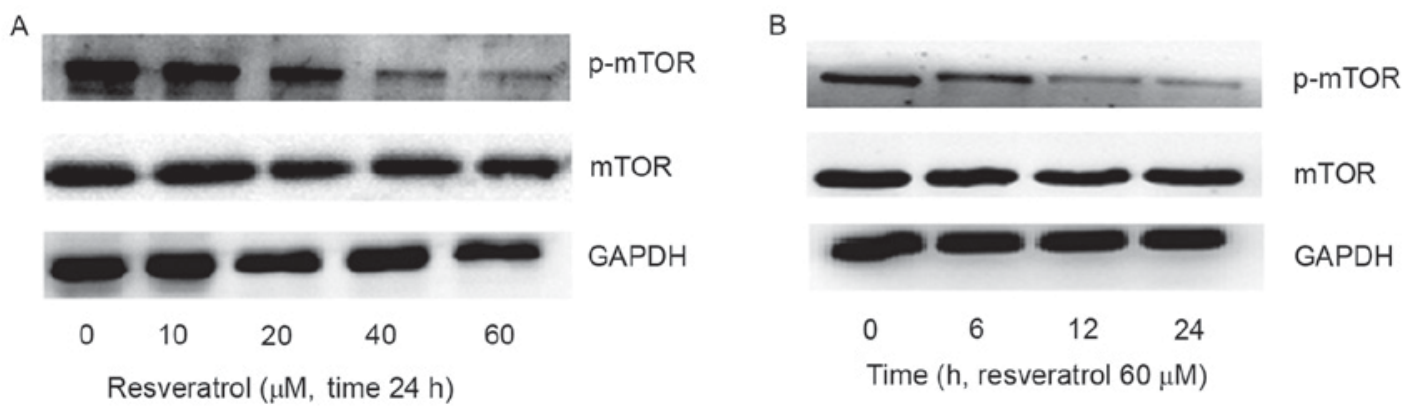

Figure 3. Resveratrol suppresses the phosphorylation of mTOR and its downstream targets in MM1.S cells. Levels of p-mTOR in MM1.S cells treated with (A) various concentrations of resveratrol for $24 \mathrm{~h}$ and (B) $60 \mu \mathrm{M}$ resveratrol for different time periods (prior to being lysed), as determined by western blot analysis. mTOR, mammalian target of rapamycin; p, phosphorylated.

$\mathrm{Ser}^{2448}$, while mTORC2 predominantly contains mTOR phosphorylated at $\operatorname{Ser}^{2481}(33)$. Western blot analysis revealed that p-mTOR Ser ${ }^{2481}$ was present in MM1.S cells, while p-mTOR $\mathrm{Ser}^{2448}$ was expressed in the two MM cell lines (Fig. 2). Subsequently, the expression of mTOR phosphorylated at $\mathrm{Ser}^{2448}$ and $\mathrm{Ser}^{2481}$ was compared with TORC1 and TORC2 readouts, respectively. The best-characterized $\mathrm{mTORC1}$ readouts are P70S6K and 4EBP, whereas the best-characterized mTORC2 readouts are Akt. The results of the present study demonstrated that baseline expression of p-mTOR $\left(\operatorname{Ser}^{2448}\right)$ is associated with TORC1-phosphorylatable P70S6K and 4EBP, and that expression of p-mTOR $\mathrm{Ser}^{2481}$ is associated with TORC2-phosphorylatable Akt Ser ${ }^{473}$ in the MM1.S cell line. p-mTOR $\operatorname{Ser}^{2448}$ is highly expressed in the RPMI-8226/DOX40 cell line, while p-Akt $\mathrm{Ser}^{473}$ is not. These results indicated that aberrant activation of mTORC1 and mTORC 2 signaling exists in the MM1.S cell line used in the present study.

Resveratrol downregulates mTOR protein expression in MM1.S cells. Analysis of the expression of the proteins involved in the $\mathrm{PI} 3 \mathrm{~K} / \mathrm{Akt} / \mathrm{mTOR}$ signaling pathway revealed that resveratrol reduced the expression of $\mathrm{p}-\mathrm{mTOR}$ in a dose-dependent manner (Fig. 3A). Similarly, the MM1.S cells were subsequently treated with $60 \mu \mathrm{M}$ resveratrol for $0,6,12$ and $24 \mathrm{~h}$ and $\mathrm{p}$-mTOR expression decreased following resveratrol treatment for $12 \mathrm{~h}$ (Fig. 3B). These results indicated that the specific inhibitor of mTOR enhances the effect induced by resveratrol.

Combined treatment with resveratrol and rapamycin is synergistic in MM1.S cell line. Following treatment with resveratrol, rapamycin, a combination of the two or DMSO for $24 \mathrm{~h}$, the MM1.S cells were collected by trypsinization and were used in western blot analysis or stored at $-80^{\circ} \mathrm{C}$ until further use. Phosphorylation of Akt was measured at $\mathrm{Ser}^{473}$ (mTORC2 substrate), phosphorylation of P70S6K was measured at $\mathrm{Thr}^{389}$ (mTORC1 substrate) and phosphorylation of 4EBP1 was measured at $\mathrm{Ser}^{65}$ (mTORC1 substrate). As demonstrated in Fig. 4A, combined treatment effectively inhibited p-Akt Ser ${ }^{473}$, p-P70S6K Thr ${ }^{389}$ and p-4EBP1 Ser ${ }^{65}$ in MM1.S cells, suggesting that combined treatment inhibits mTORC1 and mTORC2 signaling in the MM1.S cell line. However, rapamycin markedly inhibited p-P70S6K Thr ${ }^{389}$ and p-4EBP1 $\mathrm{Ser}^{65}$, but did not result in the inhibition of p-Akt $\mathrm{Ser}^{473}$, suggesting that rapamycin only inhibits mTORC1 signaling and is unable to suppress mTORC2 signaling in MM1.S cells. In addition, rapamycin treatment resulted in a significant increase in p-Akt $\mathrm{Ser}^{473}$, demonstrating that rapamycin treatment induced feedback activation of Akt signaling in MM1.S cells. Notably, resveratrol treatment effectively inhibited p-Akt $\mathrm{Ser}^{473}$, p-P70S6K Thr ${ }^{389}$ and p-4EBP1 Ser ${ }^{65}$ in MM1.S cells, indicating that resveratrol may inhibit mTORC2 and mTORC1 signaling in MM1.S cell lines.

Resveratrol, rapamycin and a combination of the two induce activation of caspase-3 and PARP in MM1.S cells. The aforementioned results demonstrated that combining resveratrol with rapamycin may inhibit mTOR signaling in MM1.S cells. In order to analyze whether treatments with rapamycin, resveratrol or a combination of the two had an effect on the apoptosis of MM1.S cells, cleavage of PARP and caspase-3, the key mediators of the apoptotic process, was detected by western blot analysis. As demonstrated in Fig. 4B, rapamycin 
A

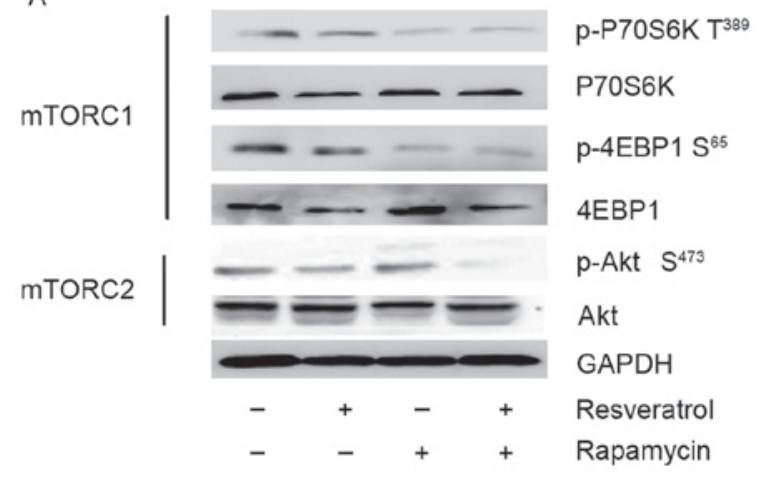

B

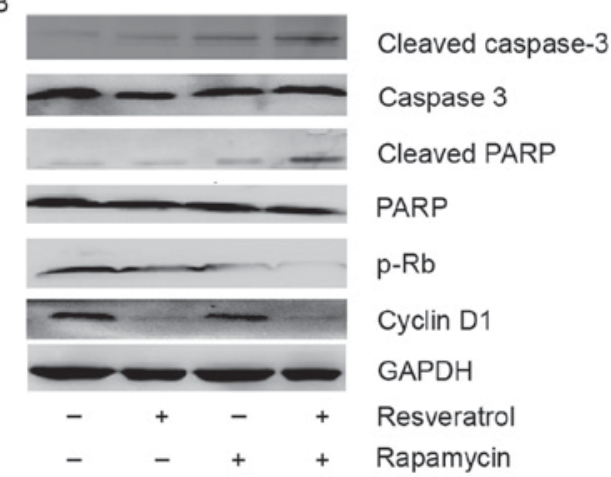

Figure 4. (A) Resveratrol, rapamycin and a combination of the two inhibit mTORC1 and mTORC2 signaling in the MM1.S cell line, as determined by immunoblot analysis. (B) Immunoblot analysis for caspase-3, PARP, p-Rb and cyclin D1 in MM1.S cells treated with resveratrol, rapamycin or a combination of the two. mTORC, mammalian transcriptional coactivator for CREB; PARP, poly (ADP-ribose) polymerase; p-Rb, phosphorylated retinoblastoma protein; Akt, protein kinase B.

induced gentle cleavage of PARP and caspase-3, consistent with its cytostatic effect. Resveratrol induced minimal cleavage of PARP and caspase-3, whereas the combination of rapamycin and resveratrol induced a higher degree of PARP and caspase-3 cleavage compared with the effect observed with each agent alone. Furthermore, the results demonstrated that PARP had undergone cleavage while the expression of caspase-3 diminished. The expression of these proteins following combined treatment decreased markedly. The results of the present study indicated that resveratrol-, rapamycin- and combined therapy-induced apoptosis in MM1.S cells is associated with caspase-3 and PARP.

Resveratrol combined with rapamycin suppresses cyclin DI expression and Rb phosphorylation in MMI.S cells. In order to further investigate the synergistic mechanism of combining resveratrol with rapamycin, the present study examined the effect of these agents on cyclin D1/Rb expression. As demonstrated in Fig. 4B, cyclin D1 and phosphorylated Rb $(\mathrm{p}-\mathrm{Rb})$ were detected in MM1.S cells treated with rapamycin, resveratrol or a combination of the two, using immunoblotting. In MM1.S cells, monotherapy with resveratrol inhibited cyclin D1 protein expression, while rapamycin barely inhibited cyclin D1 expression. Cyclins interact with cyclin-dependent kinases (CDKs) to phosphorylate Rb. Since D-cyclins are major determinants of CDK4/6 holoenzyme activity, decreased expression of cyclin D1 may prevent Rb phosphorylation in an Akt-dependent fashion. In order to confirm this hypothesis, the $\mathrm{Rb}$ phosphorylation status in MM1.S cells treated with rapamycin, resveratrol or a combination of the two was also detected. The results indicated that the combination of rapamycin and resveratrol induced downregulation of phosphorylated Rb.

\section{Discussion}

Combinations of novel agents that target various signaling pathways in MM are required in order to overcome resistance to treatment and to achieve higher response and survival rates in patients with MM. A previous study demonstrated that the combination of rapamycin with other agents, including 17-AAG, CC-5013 and dexamethasone, leads to a synergistic effect on MM cells and sensitizes the cells to apoptosis (34). Resveratrol is a naturally occurring phytoalexin and a polyphenolic compound. The molecule was revealed to be present in various fruits and vegetables and is abundant in grapes and red wine $(24,25)$. Previous studies have demonstrated that resveratrol not only has no known cytotoxicity in animal models, but also is relatively pharmacologically safe in humans $(35,36)$. Although resveratrol has been reported to have a wide range of potential targets during the inhibition of proliferation and the induction of apoptosis in a variety of tumor cell types (37), the underlying molecular mechanisms of its anticancer effects are not well understood, particularly in MM which is often difficult to treat as multiple signaling pathways are involved (38). Contradictory results have previously been reported regarding the inhibition of proliferation and the induction of apoptosis resulting from treatment with resveratrol. A previous study reported that resveratrol treatment induces apoptosis in various tumor cells (39). However, an additional study reported that resveratrol induces differentiation, but not apoptosis, in certain types of cancer cells (40). In the present study, it was revealed that the combination of rapamycin and resveratrol, tested at pharmacologically achievable doses, resulted in a strong synergistic effect on MM cell viability in vitro. This combination led to the viability impairment of MM cells and the induction of apoptosis in the two MM cell lines. However, the effects on MM1.S cells treated with a combination of rapamycin and resveratrol were more significant than the effects on the RPMI-DOX40 cells and therefore, the focus was primarily on the effect of combined treatment on MM1.S cells.

There is a large volume of evidence supporting the critical function of the PI3K/Akt/mTOR signaling pathway in cancer proliferation, tumor genesis and metastasis, and numerous studies have demonstrated that PI3K $\_$Akt_mTOR activity is increased in a variety of tumor cell lines, including MM cells (41-43). Dysregulation of mTOR function via physiological or mutational activation of upstream pathways is a common event in tumors. mTOR activation has been observed in numerous types of cancer and therefore, mTOR has become an attractive target for cancer therapy (7). As a catalytic subunit, mTOR exists in two complexes, mTORC1 $\left(\mathrm{Ser}^{2448}\right)$ and mTORC2 $\left(\mathrm{Ser}^{2481}\right)$, which function as specific scaffold 
proteins. Therefore, decreasing the expression of these two proteins may lead to reduced mTOR activity as mTOR activity is dependent upon the integrity of the complex. Impairment of any single component of the mTOR complex may reduce mTOR activity. The results of the present study demonstrated that $\mathrm{p}$-mTOR $\mathrm{Ser}^{2481}$ was present in MM1.S cells, while p-mTOR Ser ${ }^{2448}$ was revealed to be expressed in the two MM cell lines by western blot analysis (Fig. 2), indicating that the mTORC1 and mTORC2 signaling pathways were activated in the MM1.S cell lines. Rapamycin inhibits basal p70S6K activity and induces dephosphorylation of P70S6K and 4EBP-1, thereby inhibiting cap structure-dependent protein synthesis and cell proliferation (44), indicating that rapamycin may directly inhibit mTORC1 and lead to tumor cell apoptosis. Furthermore, the results of a previous study demonstrated that mTORC2 is rapamycin-insensitive, although it may be inhibited by prolonged rapamycin treatment (45). Activated mTORC1 phosphorylates P70S6K and 4EBP1, two best-characterized substrates of mTORC1, to positively regulate the protein synthesis that drives cell growth and proliferation. Therefore, $\mathrm{p}-\mathrm{P} 70 \mathrm{~S} 6 \mathrm{~K}$ and $\mathrm{p}-4 \mathrm{EBP} 1$ are frequently used in research as biomarkers of mTORC1 activity. mTORC2 has been considered to function primarily through activating Akt by phosphorylating it at $\mathrm{Ser}^{473}$, which is important for its maximal activation (33). Therefore, the level of p-Akt $\mathrm{Ser}^{473}$ is widely used to measure mTORC2 activity. The results of the present study demonstrated that the mTOR inhibitor, rapamycin, may inhibit mTORC1 signaling as p-P70S6K Thr ${ }^{389}$ and p-4EBP1 Ser ${ }^{65}$ levels were reduced, and may be unable to effectively inhibit mTORC2 signaling and result in feedback activation of Akt signaling as p-Akt $\mathrm{Ser}^{473}$ levels increased, thereby attenuating antitumor activity in MM1.S cells (Fig. 4A). The results of the present study were consistent with those of a previous study (19). By contrast, resveratrol treatment significantly decreased the phosphorylation of Akt at $\mathrm{Ser}^{473}$ in MM1.S cells, indicating that resveratrol is effective in attenuating $\mathrm{mTORC} 2$ function and preventing the feedback activation of Akt. These results indicated that resveratrol may inhibit mTORC2 signaling and may not suppress mTORC1 signaling in MM1.S cells. Therefore, combined treatment with rapamycin and resveratrol effectively inhibited p-Akt Ser ${ }^{473}$, p-P70S6K Thr ${ }^{389}$ and p-4EBP1 Ser ${ }^{65}$ in MM1.S cells, suggesting that combined treatment inhibited mTORC1 and mTORC2 signaling in the MM1.S cells.

The results of the present study demonstrated that the combination of rapamycin and resveratrol induced a significant effect on the cleavage of apoptosis-associated proteins, including PARP and caspase-3. The downstream signaling cascades targeted by rapamycin and resveratrol were subsequently delineated. The results of the present study demonstrated that rapamycin induced gentle cleavage of PARP and caspase- 3 , and that resveratrol induced minimal cleavage of PARP and caspase-3, whereas the combination of rapamycin and resveratrol induced a higher degree of PARP and caspase-3 cleavage compared with the effect observed following monotherapy with each agent alone. Furthermore, the results also demonstrated that PARP had undergone cleavage as the expression of caspase-3 decreased. Additionally, the expression of these proteins following combined treatment decreased markedly. The results of the present study indicated that resveratrol-, rapamycin- and combined therapy-induced apoptosis of MM1.S cells may be associated with caspase-3 and PARP.

In the present study, cyclin D1 and phosphorylated Rb were revealed to be inhibited in MM1.S cells treated with rapamycin and resveratrol, indicating another possible mechanism of synergy. A previous study demonstrated that low Akt activity in MM cell lines induced resistance to rapamycin by allowing continued cap-independent protein synthesis of cyclin D1 (46). In the present study, it was demonstrated that cyclin D1 was downregulated in response to resveratrol in the MM1.S cell line, indicating that cyclin D1 may be a useful marker of response to resveratrol in MM. Notably, phosphorylated Rb, another G1 regulatory protein downstream of cyclin D1, was inhibited in response to rapamycin, resveratrol and a combination of the two agents in MM1.S cells. Further studies are required to investigate the role of other cyclins and CDKs in the inhibition of phosphorylated $\mathrm{Rb}$ in cell lines with lower Akt activity. These results suggested mechanisms for synergy of rapamycin and resveratrol through targeting of multiple proteins of the PI3K/Akt/mTOR pathway and G1 regulatory proteins.

In conclusion, the results of the present study suggested that resveratrol is well-tolerated and exhibits low toxicity and is therefore safe for preclinical research. Notably, it was revealed that the newly-developed compound, resveratrol, may inhibit mTORC2 signaling. Additionally, the combination of rapamycin with resveratrol is safe and effective in inhibiting the proliferation and increasing the apoptosis of MM1.S cells. Taken together, the results of the present study indicated that combined therapy with rapamycin and resveratrol may be an attractive approach for the treatment and prevention of MM. However, these observations require further investigation in preclinical trials.

\section{Acknowledgements}

Not applicable.

\section{Funding}

No funding was received.

\section{Availability of data and materials}

The datasets used and/or analyzed during the current study are available from the corresponding author on reasonable request.

\section{Authors' contributions}

YGB made contributions to experimental design, revised the manuscript critically for important intellectual content and provided final approval of the version to be published; HGJ made substantial contributions to the acquisition of data, and analysis and interpretation of data, and was involved in drafting the manuscript. GZW was involved in performing the experiments. GHW conducted the MTT assays and analyzed cell viability. GHW was also accountable for all aspects of the work in ensuring that questions related to the accuracy or integrity of any part of the work are appropriately resolved. All authors read and approved the final manuscript. 


\section{Ethics approval and consent to participate}

This experiment only involves cell level research, approved by Ethics Committee of Affiliated Hospital of Inner Mongolia University for the Nationalities (Ethics Committee no. 0010/2016).

\section{Consent for publication}

Not applicable.

\section{Competing interests}

The authors declare that they have no competing interests.

\section{References}

1. Palumbo A and Anderson K: Multiple myeloma. N Engl J Med 364: 1046-1060, 2011

2. Kyle RA, Gertz MA, Witzig TE, Lust JA, Lacy MQ, Dispenzieri A, Fonseca R, Rajkumar SV, Offord JR, Larson DR, et al: Review of 1027 patients with newly diagnosed multiple myeloma. Mayo Clin Proc 78: 21-33, 2003.

3. Cao B, Li J and Mao X: Dissecting bortezomib: Development, application, adverse effects and future direction. Curr Pharm Des 19: 3190-3200, 2013.

4. Herndon TM, Deisseroth A, Kaminskas E, Kane RC, Koti KM, Rothmann MD, Habtemariam B, Bullock J, Bray JD, Hawes J, et al: U.S. Food and drug administration approval: Carfilzomib for the treatment of multiple myeloma. Clin Cancer Res 19: 4559-4563, 2013.

5. Wang F, Zhang W, Guo L, Bao W, Jin N, Liu R, Liu P, Wang Y, Guo Q and Chen B: Gambogic acid suppresses hypoxiainduced hypoxia-inducible factor- $1 \alpha /$ vascular endothelial growth factor expression via inhibiting phosphatidylinositol 3-kinase/Akt/mammalian target protein of rapamycin pathway in multiple myeloma cells. Cancer Sci 105: 1063-1070, 2014.

6. Adachi M, Hoshino Y, Izumi Y, Sakai H and Takagi S: Effects of inhibitors of vascular endothelial growth factor receptor 2 and downstream pathways of receptor tyrosine kinases involving phosphatidylinositol 3-kinase/Akt/mammalian target of rapamycin or mitogen-activated protein kinase in canine hemangiosarcoma cell lines. Can J Vet Res 80: 209-216, 2016.

7. Cirstea D, Santo L, Hideshima T, Eda H, Mishima Y, Nemani N, Mahindra A, Yee A, Gorgun G, Hu Y, et al: Delineating the mTOR Kinase pathway using a dual TORC1/2 inhibitor, AZD8055, in multiple myeloma. Mol Cancer Ther 13: 2489-2500, 2014.

8. Gera $\mathbf{J}$ and Lichtenstein A: The mammalian target of rapamycin pathway as a therapeutic target in multiple myeloma. Leuk Lymphoma 52: 1857-1866, 2011.

9. Dowling RJ,Topisirovic I,Fonseca BD and Sonemberg N: Dissecting the role of mTOR: Lessons from mTOR inhibitor. Biochim Biophys Acta 1804: 433-439, 2010.

10. Laplante M and Sabatini DM: mTOR signaling in growth control and disease. Cell 149: 274-293, 2010.

11. Hara K, Yonezawa K, Weng QP, Kozlowski MT, Belham C and Avruch J: Amino acid sufficiency and mTOR regulate p70 S6 kinase and eIF-4E BP1 through a common effector mechanism. J Biol Chem 273: 14484-14494, 1998.

12. Sarbassov DD, Guertin DA, Ali SM and Sabatini DM: Phosphorylation and regulation of Akt/PKB by the rictor-mTOR complex. Science 307: 1098-1101, 2005.

13. Serrano M: Dissecting the role of mTOR complexes in cellular senescence. Cell Cycle 11: 2231-2232, 2012.

14. Lang SA, Hackl C, Moser C, Fichtner-Feigl S, Koehl GE, Schlitt HJ, Geissler EK and Stoeltzing O: Implication of RICTOR in the mTOR inhibitor-mediated induction of insulin-like growth factor-I receptor (IGF-IR) and human epidermal growth factor receptor-2 (Her2) expression in gastrointestinal cancer cells. Biochim Biophys Acta 1803: 435-442, 2010.

15. Wander SA, Hennessy BT and Slingerland JM: Next-generation mTOR inhibitors in clinical oncology: How pathway complexity informs therapeutic strategy. J Clin Invest 121: 1231-1241, 2011.
16. Li J, Zhu J, Cao B and Mao X: The mTOR signaling pathway is an emerging therapeutic target in multiple myeloma. Curr Pharm Des 20: 125-135, 2014.

17. Willems L, Tamburini J, Chapuis N, Lacombe C, Mayeux P and Bouscary D: PI3K and mTOR signaling pathways in cancer: New data on targeted therapies. Curr Oncol Rep 14: 129-138, 2012.

18. Benjamin D, Colombi M, Moroni C and Hall MN: Rapamycin passes the torch: A new generation of mTOR inhibitors. Nat Rev Drug Discov 10: 868-880, 2011.

19. Feldman ME, Apsel B, Uotila A, Loewith R, Knight ZA, Ruggero D and Shokat KM: Active-site inhibitors of mTOR target rapamycin-resistant outputs of mTORC1 and mTORC2. PLoS Biol 7: e38, 2009.

20. Liu DM, Zhao L, Liu TT, Jiao PL, Zhao DD, Shih MS, Tao B, Sun LH, Zhao HY and Liu JM: Rictor/mTORC2 loss in osteoblasts impairs bone mass and strength. Bone 90: 50-58, 2016.

21. Hsieh AC, Costa M, Zollo O, Davis C, Feldman ME, Testa JR, Meyuhas O, Shokat KM and Ruggero D: Genetic dissection of the oncogenic mTOR pathway reveals druggable addiction to translational control via 4EBP-eIF4E. Cancer Cell 17: 249-261, 2010.

22. Yan H, Frost P, Shi Y, Hoang B, Sharma S, Fisher M, Gera J and Lichtenstein A: Mechanism by which mammalian target of rapamycin inhibitors sensitize multiple myeloma cells to dexamethasone-induced apoptosis. Cancer Res 66: 2305-2313, 2006.

23. Francis LK, Alsayed Y, Leleu X, Jia X, Singha UK, Anderson J, Timm M, Ngo H, Lu G, Huston A, et al: Combination mammalian target of rapamycin inhibitor rapamycin and HSP90 inhibitor 17-allylamino-17-demetoxiygeldanamycin has synergistic activity in multiple myeloma. Clin Cancer Res 12: 6826-6835, 2006.

24. Gupta SC, Kannappan R, Reuter S, Kim JH and Aggarwal BB: Chemosensitization of tumors by resveratrol. Ann N Y Acad Sci 1215: 150-160, 2011.

25. Baur JA and Sinclair DA: Therapeutic potential of resveratrol: The in vivo evidence. Nat Rev Drug Discov 5: 493-506, 2006.

26. Bishayee A: Cancer prevention and treatment with resveratrol: From rodent studies to clinical trials. Cancer Prev Res (Phila) 2: 409-418, 2009.

27. Bhardwaj A, Sethi G, Vadhan-Raj S, Bueso-Ramos C, Takada Y, Gaur U, Nair AS, Shishodia S and Aggarwal BB: Resveratrol inhibits proliferation, induces apoptosis, and overcomes chemoresistance through down-regulation of STAT3 and nuclear factor-kappaB-regulated antiapoptotic and cell survival gene products in human multiple myeloma cells. Blood 109: 2293-2302, 2007.

28. Boissy P, Andersen TL, Abdallah BM, Kassem M, Plesner T and Delaissé JM: Resveratrol inhibits myeloma cell growth, prevents osteoclast formation, and promotes osteoblast differentiation. Cancer Res 65: 9943-9952, 2005.

29. Kueck A, Opipari AW Jr, Griffith K, Tan L, Choi M, Huang J, Wahl H and Liu JR: Resveratrol inhibits glucose metabolism in human ovarian cancer cells. Gynecol Oncol 107: 450-457, 2007.

30. Waite KA, Sinden MR and Eng C: Phytoestrogen exposure elevates PTEN levels. Hum Mol Genet 14: 1457-1463, 2005.

31. He X, Wang Y,Zhu J, Orloff Mand Eng C: Resveratrol enhances the anti-tumor activity of the mTOR inhibitor rapamycin in multiple breast cancer cell lines mainly by suppressing rapamycin-induced AKT signaling. Cancer Lett 301: 168-176, 2011.

32. Sui T, Ma L, Bai X, Li Q and Xu X: Resveratrol inhibits the phosphatidylinositide 3-kinase/protein kinase $\mathrm{B} / \mathrm{mammalian}$ target of rapamycin signaling pathway in the human chronic myeloid leukemia K562 cell line. Oncol Lett 7: 2093-2098, 2014.

33. Copp J, Manning G and Hunter T: TORC-specific phosphorylation of mammalian target of rapamycin (mTOR): Phospho-Ser2481 is a marker for intact mTOR signaling complex 2. Cancer Res 69: 1821-1827, 2009

34. Raje N, Kumar S, Hideshima T, Ishitsuka K, Chauhan D, Mitsiades C,Podar K, Le Gouill S, Richardson P, Munshi NC, et al: Combination of the mTOR inhibitor rapamycin and CC-5013 has synergistic activity in multiple myeloma. Blood 104: 4188-4193, 2004.

35. Cao Y, Fu ZD, Wang F, Liu HY and Han R: Anti-angiogenic activity of resveratrol, a natural compound from medicinal plants. J Asian Nat Prod Res 7: 205-213, 2005.

36. Gusman J, Malonne $\mathrm{H}$ and Atassi G: A reappraisal of the potential chemopreventive and chemotherapeutic properties of resveratrol. Carcinogenesis 22: 1111-1117, 2001.

37. Wang B, Liu J and Gong Z: Resveratrol induces apoptosis in K562 cells via the regulation of mitochondrial signaling pathways. Int J Clin Exp Med 8: 16926-16933, 2015. 
38. Jang J, Jeong SJ, Kwon HY, Jung JH, Sohn EJ, Lee HJ, Kim JH, Kim SH, Kim JH and Kim SH: Decursin and doxorubicin are in synergy for the induction of apoptosis via STAT3 and/or mTOR pathways in human multiple myeloma cells. Evid Based Complement Alternat Med 2013: 506324, 2013.

39. Quoc Trung L, Espinoza JL, Takami A and Nakao S: Resveratrol induces cell cycle arrest and apoptosis in malignant NK cells via JAK2/STAT3 pathway inhibition. PLoS One 8: e55183, 2013.

40. Chen Q, Ganapathy S, Singh KP, Shankar S and Srivastava RK Resveratrol induces growth arrest and apoptosis through activation of FOXO transcription factors in prostate cancer cells. PLoS One 5: e15288, 2010.

41. Li H, Hu J, Wu S, Wang L, Cao X, Zhang X, Dai B, Cao M, Shao R, ZhangR,etal:Auranofin-mediatedinhibition of PI3K/AKT/mTOR axis and anticancer activity in non-small cell lung cancer cells Oncotarget 7: 3548-3558, 2016.

42. Que W, Chen J, Chuang M and Jiang D: Knockdown of c-Met enhances sensitivity to bortezomib in human multiple myeloma U266 cells via inhibiting Akt/mTOR activity. APMIS 120 195-203, 2012.

43. Feng N, Luo J and Guo X: Silybin suppresses cell proliferation and induces apoptosis of multiple myeloma cells via the PI3K/Akt/mTOR signaling pathway. Mol Med Rep 13: 3243-3248, 2016.
44. Rousseau D, Gingras AC, Pause A and Sonenberg N: The eIF4E-binding proteins 1 and 2 are negative regulators of cell growth. Oncogene 13: 2415-2420, 1996

45. Sarbassov DD, Ali SM, Kim DH, Guertin DA, Latek RR, Erdjument-Bromage H, Tempst P and Sabatini DM: Rictor, a novel binding partner of mTOR, defines a rapamycin-insensitive and raptor-independent pathway that regulates the cytoskeleton. Curr Biol 14: 1296-1302, 2004.

46. Gera JF, Mellinghoff IK, Shi Y, Rettig MB, Tran C, Hsu JH, Sawyers CL and Lichtenstein AK: AKT activity determines sensitivity tomammalian target of rapamycin (mTOR) inhibitors by regulating cyclin D1 and c-myc expression. J Biol Chem 279: 2737-2746, 2004 